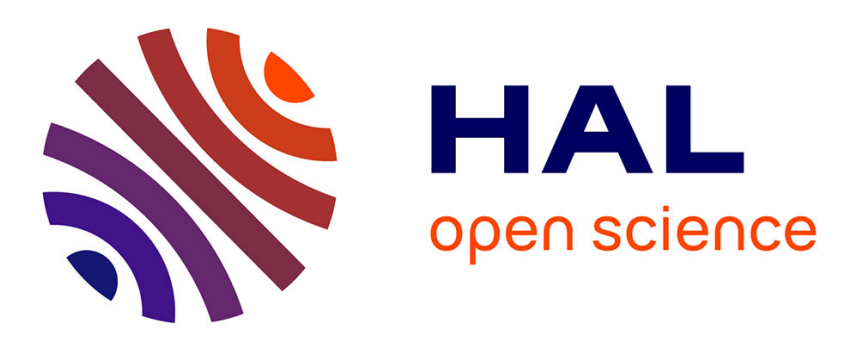

\title{
Uncertainty quantification in low-frequency dynamics of complex beam-like structures having a high-modal density
}

\author{
Anas Batou, Christian Soize
}

\section{- To cite this version:}

Anas Batou, Christian Soize. Uncertainty quantification in low-frequency dynamics of complex beamlike structures having a high-modal density. International Journal for Uncertainty Quantification, 2013, 3 (6), pp.475-485. 10.1615/Int.J.UncertaintyQuantification.2012005286 . hal-00808866

\section{HAL Id: hal-00808866 https://hal.science/hal-00808866}

Submitted on 8 Apr 2013

HAL is a multi-disciplinary open access archive for the deposit and dissemination of scientific research documents, whether they are published or not. The documents may come from teaching and research institutions in France or abroad, or from public or private research centers.
L'archive ouverte pluridisciplinaire HAL, est destinée au dépôt et à la diffusion de documents scientifiques de niveau recherche, publiés ou non, émanant des établissements d'enseignement et de recherche français ou étrangers, des laboratoires publics ou privés. 


\title{
UNCERTAINTY QUANTIFICATION IN LOW- FREQUENCY DYNAMICS OF COMPLEX BEAM-LIKE STRUCTURES HAVING A HIGH-MODAL DENSITY
}

\author{
Anas Batou* E Christian Soize \\ Universit Paris-Est, Laboratoire Modlisation et Simulation Multi Echelle, MSME UMR 8208 \\ CNRS, 5 bd Descartes, 77454 Marne-la-Valle, France \\ Original Manuscript Submitted: 08/25/2012; Final Draft Received: 08/25/2012 \\ The paper deals with the construction of a stochastic reduced-order model for beam-like dynamical structures having a \\ high modal density in the low-frequency range for which the classical methods used to construct a reduced-order model \\ are not adapted. We then use a method recently proposed which consists in constructing a basis of the global displace- \\ ments and a basis of the local displacements by solving two unusual eigenvalue problems. The stochastic reduced-order \\ model is then construct using the basis of the global displacements. The contribution of the local displacements is \\ taken into account in the reduced-order model using a statistical approach. The theory is presented and is applied to \\ a computational model of fuel assemblies for which the dynamical response must be characterized in terms of global \\ displacements.
}

KEY WORDS: Reduced-order model, structural dynamics; beam-like structures, uncertainty quantification, UQ, global displacements, local displacements; vibrations;

\section{INTRODUCTION}

This paper is devoted to the construction of a stochastic reduced-order model (ROM) for complex beam-like dynamical structures having a high modal density in the low-frequency (LF) range. A beam-like structure can be defined as a slender structure for which, in the low-frequency band, the dynamical behavior is dominated by global transverse bending elastic modes, but for which there are also, in the same frequency band, local elastic modes. For instance a fuel assembly of pressurized reactor (see Application) is a beam-like structure, but such structures can be found in the naval industry (tankers, large ships), in space industry (launchers, space orbital station), etc. The LF range is classically

\footnotetext{
${ }^{*}$ Correspond to: Anas Batou, E-mail: anas.batou@univ-paris-est.fr
} 
characterized by a very low modal density, which means that the resonances are well separated. In this case, classical methods (such as modal analysis see $[1,2]$ ) can be used in order to construct a ROM with a small dimension which allows the LF dynamical responses to be efficiently predicted with a good accuracy and a good robustness with respect to both the system parameters uncertainties and the model uncertainties induced by modeling errors. Furthermore, in this case, the ROM can easily be updated using experimental modal analysis techniques and experimental responses [3, 4]. In general, this low-frequency range can clearly be separated from the medium-frequency range and from the highfrequency range for which the modal density is larger and for which adapted methods have to be introduced in order to construct efficient ROMs [2, 5]. In this paper, we are interested in the dynamical analysis of complex beam-like structures constituted of a master stiff structure for which its displacements are mainly global and of several flexible substructures for which their displacements are mainly local. The eigenfrequencies of the first local elastic modes of each free-free flexible substructure (not connected to the master structure) belong to the same LF band as the first global elastic modes of the master stiff structure. As a consequence, as soon as the flexible substructures are connected to the master structure, the local elastic modes induce numerous local resonances in the LF range. Furthermore, due to this coupling between the master structure and the flexible substructures, there are some couplings between the global displacements and the local displacements. The deformations of global elastic modes are mainly composed of global displacements but also of local displacements contributions due to local elastic modes. Consequently, when there are numerous local elastic modes in the LF ranges, the elastic modes cannot easily be separated into global elastic modes and local elastic modes. There are no efficient sorting method which could be used to select the elastic modes in terms of global elastic modes and local elastic modes. In addition, although the reduced-order model must be constructed with respect to the global elastic modes, this ROM must have the capability to predict the amplitudes of the responses of the structure in this LF range. For an excitation applied to the master structure, since there are local elastic modes in this LF frequency band, a part of the mechanical energy of the responses of the master structure is transferred from the generalized coordinates associated with the global elastic modes to the generalized coordinates associated with the local elastic modes which store this energy and then induce an apparent damping at the resonances associated with the global coordinates [6].

Recently, a new methodology for the construction of a ROM adapted to the LF range in presence of numerous local elastic modes has been introduced [7]. This method introduces two unusual eigenvalue problems for which the solutions do not correspond to the classical elastic modes. They provide a basis of the global displacements space and a basis of the local displacements space. It is proven that the union of these two bases yields a basis of the admissible displacements space which can be written as the direct sum of the global displacements space and of the 
local displacements space. The first step of this method consists in decomposing the domain of the structure into non-overlapping subdomains. The choice of the geometry of the subdomains allows the characterization of the global displacements space to be controlled. A projection operator is constructed on the base of the decomposition in subdomains and is defined as a kinematic reduction on each subdomain. For the construction of the global displacements space, the proposed methodology corresponds to a reduction of the kinetic energy while the elastic energy is kept exact.

Global displacements are generally robust with respect to uncertainties (except uncertainties induced by modeling errors of the boundary conditions) while local displacements are very sensitive to both the system parameter uncertainties and model uncertainties induced by modeling errors. The introduction of the generalized coordinates associated with the basis of the global (or the local) displacements space, briefly called the global (or the local) generalized coordinates, proposed in [7], provides a practical frame which allows us to construct a stochastic ROM for the dynamical system under consideration. It consists in implementing a probabilistic model of uncertainties only related to the local generalized coordinates.

Concerning the previous researches relative to the construction of a ROM for complex beam-like structures having a high-modal density in the LF range, most of them are based on the introduction of an equivalent beam model [8,9] or on the use of homogenization methods [10]. The development of such simplified models requires an expertise concerning the dynamical behavior for this type of complex beam-like structure and provide a quite good approximation of the dynamical responses in the LF range in terms of the global displacements. However, these approximations do not allow the local contributions to be taken into account. Furthermore, the construction of an accurate simplified model cannot automatically be carried out and a procedure of validation of the simplified model is always needed. In [11], the authors propose to construct a basis of the global displacements space using a rough finite element model. Nevertheless, such a rough finite element model does not allow a good approximation of the elastic energy to be obtained for heterogeneous structures with complex geometries. The Proper Orthogonal Decomposition (POD) method (see [12-14]) allows in some cases to extract an accurate small size basis in order to construct a ROM, but this basis has to be constructed a posteriori, which means that a sufficiently rich dynamical response must be constructed. Moreover, the usual POD basis is only optimal for a given external load (or imposed displacement) while the POD method proposed for the linear dynamical systems in [4] is optimal for any external loads (or any imposed displacements).

The objective of this paper is double. The first one consists in constructing a ROM in the LF range for complex beam-like dynamical structures having a high-modal density in the LF range. The second one is to take into account the effects of the local elastic modes on the ROM in this LF range. The first objective is achieved using the method 
introduced above for which a subdomains decomposition of the domain is proposed. The second objective is achieved introducing a probabilistic model of uncertainties for the local generalized coordinates as explained above the stochastic modeling which is proposed differs from the one introduced in [7]. Note that the objective is not to construct a reduced-order model which would be of smaller size than a reduced-order model constructed with a classical modal analysis, but is to separate the global contributions from the local contributions, and to model each of this contribution in different ways with respect to uncertainties.

Section 2 is devoted to the introduction of the computational dynamical model and its usual ROM constructed with the elastic modes. In Section 3, the details of the methodology are summarized and an adapted subdomains decomposition of the domain is presented. Section 4 is devoted to the construction of the probabilistic model of uncertainties which allows us to statistically take into account the effects of the local elastic modes on the LF responses of the constructed ROM. In Section 5, the methodology is applied on a computational model of half fuel assembly of pressurized water reactor.

\section{COMPUTATIONAL DYNAMICAL MODEL AND ITS USUAL REDUCED-ORDER MODEL}

The computational dynamical model is constructed using the Finite Element (FE) method and its usual reduction using the elastic modes is introduced.

We are interested in predicting the frequency response functions of a three dimensional linear damped beam-like structure, occupying a bounded domain $\Omega$, in the frequency band of analysis $\mathcal{B}=\left[\omega_{\min }, \omega_{\max }\right]$ with $0<\omega_{\min }$. The complex vector $\mathbb{U}(\omega)$ of the $m$ degrees-of-freedom (DOF) of the computational model is solution of the following complex matrix equation,

$$
\left(-\omega^{2}[\mathbb{M}]+i \omega[\mathbb{D}]+[\mathbb{K}]\right) \mathbb{U}(\omega)=\mathbb{F}(\omega)
$$

in which $\omega$ is the frequency in $\operatorname{rad} / \mathrm{s}$ and $i=\sqrt{-1}$, where $[\mathbb{M}],[\mathbb{D}]$ and $[\mathbb{K}]$ are the $(m \times m)$ positive-definite symmetric real mass, damping and stiffness matrices and where $\mathbb{F}(\omega)$ corresponds to the discretization of the external forces. The eigenfrequencies and the elastic modes of the associated conservative dynamical system are determined in finding $\lambda$ and $\varphi$ in $\mathbb{R}^{m}$ such that

$$
[\mathbb{K}] \varphi=\lambda[\mathbb{M}] \varphi
$$

Using the modal analysis method, the approximation $\mathbb{U}^{(n)}(\omega)$ at order $n$ of $\mathbb{U}(\omega)$ is written as 


$$
\mathbb{U}^{(n)}(\boldsymbol{\omega})=\sum_{\alpha=1}^{n} q_{\alpha}(\omega) \boldsymbol{\varphi}_{\alpha}=[\Phi] \boldsymbol{q}(\omega),
$$

in which $\boldsymbol{q}(\omega)=\left(q_{1}(\omega), \ldots, q_{n}(\omega)\right)$ is the complex vector of the $n$ generalized coordinates and where $[\Phi]=$ $\left[\boldsymbol{\varphi}_{1} \ldots \boldsymbol{\varphi}_{n}\right]$ is the $(m \times n)$ real matrix of the elastic modes associated with the $n$ first eigenvalues. The modal analysis method allows the dynamical response of the structure to be predicted in the LF range with a good accuracy if the modal density is low, that is not the case for the dynamical structure we are interested in. However, the elastic modes and the reduced-order model obtained using the modal analysis will be used for the methodology proposed in order to construct the LF ROM for beam-like structure, using a double projection method (see Section 3.2).

\section{BASES OF THE GLOBAL AND LOCAL DISPLACEMENTS SPACES}

In this Section, the method presented in [7] for continuous and discrete cases is summarized for the discrete case (using the FE method).

\subsection{Decomposition of the mass matrix}

The first step consists in partitioning the domain $\Omega$ into $n_{J}$ non-overlapping subdomains $\Omega_{j}$ such that

$$
\Omega=\bigcup_{j=1}^{n_{J}} \Omega_{j} \quad, \quad \Omega_{j} \cap \Omega_{k}=\emptyset \text { for } j \neq k .
$$

More details concerning the subdomains composition of the domain for beam-like structures are given in Section 3.4. Let $\boldsymbol{u} \mapsto h^{r}(\boldsymbol{u})$ be the linear operator defined by

$$
\left\{h^{r}(\boldsymbol{u})\right\}(\boldsymbol{x})=\sum_{j=1}^{n_{J}} \mathbb{1}_{\Omega_{j}}(\boldsymbol{x}) \frac{1}{m_{j}} \int_{\Omega_{j}} \rho\left(\boldsymbol{x}^{\prime}\right) \boldsymbol{u}\left(\boldsymbol{x}^{\prime}\right) d \boldsymbol{x}^{\prime},
$$

in which $\boldsymbol{x} \mapsto \mathbb{1}_{\Omega_{j}}(\boldsymbol{x})=1$ if $\boldsymbol{x}$ is in $\Omega_{j}$ and $=0$ otherwise. For all $j$ in $\left\{1, \ldots, n_{J}\right\}$, the local mass $m_{j}$ is defined by $m_{j}=\int_{\Omega_{j}} \rho(\boldsymbol{x}) d \boldsymbol{x}$, where $\boldsymbol{x} \mapsto \rho(\boldsymbol{x})$ is the mass density. This operator carries out an average of the displacements with respect to the mass density in each subdomain $\Omega_{j}$ (kinematic reduction). Let $\boldsymbol{u} \mapsto h^{c}(\boldsymbol{u})$ be the linear operator defined by

$$
h^{c}(\boldsymbol{u})=\boldsymbol{u}-h^{r}(\boldsymbol{u})
$$


This operator is the complementary of operator $h^{r}$ and sets the average of the displacements with respect to the mass density to zero. Let $\left[H^{r}\right]$ be the $(m \times m)$ matrix relative to the finite element discretization of the projection operator $h^{r}$ defined by Eq. (5) and let $\left[H^{c}\right]$ be the $(m \times m)$ matrix relative to the finite element discretization of the projection operator $h^{c}$ defined by Eq. (6). We then have $\left[H^{c}\right]=\left[I_{m}\right]-\left[H^{r}\right]$, where $\left[I_{m}\right]$ is the $(m \times m)$ identity matrix. The $(m \times m)$ projected mass matrix $\left[\mathbb{M}^{r}\right]$ is such that

$$
\left[\mathbb{M}^{r}\right]=\left[H^{r}\right]^{T}[\mathbb{M}]\left[H^{r}\right]
$$

and satisfies the following property,

$$
\left[\mathbb{M}^{r}\right]=[\mathbb{M}]\left[H^{r}\right]=\left[H^{r}\right]^{T}[\mathbb{M}]
$$

The $(m \times m)$ complementary mass matrix $\left[\mathbb{M}^{c}\right]$ is such that

$$
\left[\mathbb{M}^{c}\right]=\left[H^{c}\right]^{T}[\mathbb{M}]\left[H^{c}\right]
$$

and can be written as,

$$
\left[\mathbb{M}^{c}\right]=[\mathbb{M}]-\left[\mathbb{M}^{r}\right]
$$

The projected mass matrix $\left[\mathbb{M}^{r}\right]$ and the complementary mass matrix $\left[\mathbb{M}^{c}\right]$ are useful for the construction of the global eigenvalue problem and the local eigenvalue problem defined hereinafter.

\subsection{Construction of the bases for the global and the local displacements spaces}

The basis of the global displacements space is constituted of the eigenvectors $\boldsymbol{\phi}^{g}$ in $\mathbb{R}^{m}$ of the following eigenvalue problem, called the global eigenvalue problem,

$$
[\mathbb{K}] \boldsymbol{\phi}^{g}=\lambda^{g}\left[\mathbb{M}^{r}\right] \boldsymbol{\phi}^{g},
$$

in which the stiffness matrix is kept exact while the mass matrix is projected. This generalized eigenvalue problem admits an increasing sequence of $3 n_{J}$ positive eigenvalues $0<\lambda_{1}^{g} \leq \ldots \leq \lambda_{3 n_{J}}^{g}$, associated with the algebraically independent eigenvectors $\left\{\boldsymbol{\phi}_{1}^{g}, \ldots, \boldsymbol{\phi}_{3 n_{J}}^{g}\right\}$, called the global eigenvectors, which are used as the basis of the global displacements space and which do not correspond to elastic modes. The basis of the local displacements space is made up of the eigenvectors $\phi^{\ell}$ in $\mathbb{R}^{m}$ of the following generalized eigenvalue problem, called the local eigenvalue 
problem,

$$
[\mathbb{K}] \boldsymbol{\phi}^{\ell}=\lambda^{\ell}\left[\mathbb{M}^{c}\right] \boldsymbol{\phi}^{\ell}
$$

in which the stiffness matrix is kept exact while the mass matrix is projected. This generalized eigenvalue problem admits an increasing sequence of positive eigenvalues $0<\lambda_{1}^{\ell} \leq \ldots \leq \lambda_{m-3 n_{J}}^{\ell}$, associated with the algebraically independent eigenvectors $\left\{\boldsymbol{\phi}_{1}^{\ell}, \ldots, \boldsymbol{\phi}_{m-3 n_{J}}^{\ell}\right\}$, called the local eigenvectors, which are used as the basis of the local displacements space and which do not correspond to elastic modes. In practice, the basis of the global displacements space and the basis of the local displacements space are calculated using a double projection method which is less intrusive with respect to the commercial software and less time-consuming than the direct method. For $n$ sufficiently large, the solutions of the generalized eigenvalue problems defined by Eqs. (11) and (12) are then rewritten as

$$
\boldsymbol{\phi}^{g}=[\Phi] \widetilde{\boldsymbol{\phi}}^{g} \quad, \quad \boldsymbol{\phi}^{\ell}=[\Phi] \widetilde{\boldsymbol{\phi}}^{\ell}
$$

in which $[\Phi]$ is the modal matrix defined by Eq. (3). The eigenvectors $\widetilde{\phi}^{g}$ are the solutions of the generalized eigenvalue problem

$$
[\widetilde{K}] \widetilde{\boldsymbol{\phi}}^{g}=\lambda^{g}\left[\widetilde{M}^{r}\right] \widetilde{\boldsymbol{\phi}}^{g}
$$

in which $\left[\widetilde{M}^{r}\right]=\left[\Phi^{r}\right]^{T}[\mathbb{M}]\left[\Phi^{r}\right]$ and $[\widetilde{K}]=[\Phi]^{T}[\mathbb{K}][\Phi]$, and where the $(m \times n)$ real matrix $\left[\Phi^{r}\right]$ is such that $\left[\Phi^{r}\right]=\left[H^{r}\right][\Phi]$. The eigenvectors $\tilde{\boldsymbol{\phi}}^{\ell}$ are the solutions of the generalized eigenvalue problem

$$
[\widetilde{K}] \widetilde{\boldsymbol{\phi}}^{\ell}=\lambda^{\ell}\left[\widetilde{M}^{c}\right] \widetilde{\boldsymbol{\phi}}^{\ell}
$$

in which $\left[\widetilde{M}^{c}\right]=\left[\Phi^{c}\right]^{T}[\mathbb{M}]\left[\Phi^{c}\right]$ and where the $(m \times n)$ real matrix $\left[\Phi^{c}\right]$ is such that $\left[\Phi^{c}\right]=\left[H^{c}\right][\Phi]=[\Phi]-\left[\Phi^{r}\right]$.

\subsection{Mean reduced-order model (ROM)}

In [7], it is proven that the family $\left\{\boldsymbol{\phi}_{1}^{g}, \ldots, \boldsymbol{\phi}_{3 n_{J}}^{g}, \boldsymbol{\phi}_{1}^{\ell}, \ldots, \boldsymbol{\phi}_{m-3 n_{J}}^{\ell}\right\}$ is a basis of $\mathbb{R}^{m}$. The mean reduced-order model of dimension $\left(n_{g}, n_{\ell}\right)$ is defined as the projection $\mathbb{U}_{n_{g}, n_{\ell}}(\omega)$ of $\mathbb{U}(\omega)$ on the subspace of $\mathbb{C}^{m}$ spanned by the family $\left\{\boldsymbol{\phi}_{1}^{g}, \ldots, \boldsymbol{\phi}_{n_{g}}^{g}, \boldsymbol{\phi}_{1}^{\ell}, \ldots, \boldsymbol{\phi}_{n_{\ell}}^{\ell}\right\}$ of real vectors associated with the $n_{g}$ first global eigenvectors such that $n_{g} \leq 3 n_{J} \leq m$ and with the $n_{\ell}$ first local eigenvectors such that $n_{\ell} \leq m-3 n_{J}$. Then, the approximation $\mathbb{U}_{n_{g}, n_{\ell}}(\omega)$ 
of $\mathbb{U}(\omega)$ at order $\left(n_{g}, n_{\ell}\right)$ is written as

$$
\mathbb{U}_{n_{g}, n_{\ell}}(\boldsymbol{\omega})=\sum_{\alpha=1}^{n_{g}} q_{\alpha}^{g}(\boldsymbol{\omega}) \boldsymbol{\phi}_{\alpha}^{g}+\sum_{\beta=1}^{n_{\ell}} q_{\beta}^{\ell}(\boldsymbol{\omega}) \boldsymbol{\phi}_{\beta}^{\ell}
$$

Let be $n_{t}=n_{g}+n_{\ell}$. Let $\boldsymbol{q}(\boldsymbol{\omega})=\left(\boldsymbol{q}^{g}(\boldsymbol{\omega}), \boldsymbol{q}^{\ell}(\boldsymbol{\omega})\right)$ be the vector in $\mathbb{C}^{n_{t}}$ of all the generalized coordinates such that $\boldsymbol{q}^{g}(\boldsymbol{\omega})=\left(q_{1}^{g}(\omega), \ldots, q_{n_{g}}^{g}(\omega)\right)$ and $\boldsymbol{q}^{\ell}(\boldsymbol{\omega})=\left(q_{1}^{\ell}(\omega), \ldots, q_{n_{\ell}}^{\ell}(\omega)\right)$. Consequently, vector $\boldsymbol{q}(\boldsymbol{\omega})$ is solution of the following mean reduced matrix equation,

$$
\left(-\omega^{2}[M]+i \omega[D]+[K]\right) \boldsymbol{q}(\omega)=\mathcal{F}(\omega)
$$

where $[M],[D]$ and $[K]$ are the $\left(n_{t} \times n_{t}\right)$ mean generalized mass, damping and stiffness matrices defined by blocks

as

$$
[M]=\left[\begin{array}{cc}
M^{g g} & M^{g \ell} \\
\left(M^{g \ell}\right)^{T} & M^{\ell \ell}
\end{array}\right],[D]=\left[\begin{array}{cc}
D^{g g} & D^{g \ell} \\
\left(D^{g \ell}\right)^{T} & D^{\ell \ell}
\end{array}\right],[K]=\left[\begin{array}{cc}
K^{g g} & K^{g \ell} \\
\left(K^{g \ell}\right)^{T} & K^{\ell \ell}
\end{array}\right] .
$$

Let $A$ (or $\mathbb{A}$ ) be denoting $M, D$ or $K$ (or $\mathbb{M}, \mathbb{D}$ or $\mathbb{K}$ ). Therefore, the block matrices are defined by

$$
\left[A^{g g}\right]_{\alpha \beta}=\left(\boldsymbol{\phi}_{\alpha}^{g}\right)^{T}[\mathbb{A}] \boldsymbol{\phi}_{\beta}^{g},\left[A^{g \ell}\right]_{\alpha \beta}=\left(\boldsymbol{\phi}_{\alpha}^{g}\right)^{T}[\mathbb{A}] \boldsymbol{\phi}_{\beta}^{\ell},\left[A^{\ell \ell}\right]_{\alpha \beta}=\left(\boldsymbol{\phi}_{\alpha}^{\ell}\right)^{T}[\mathbb{A}] \boldsymbol{\phi}_{\beta}^{\ell},
$$

which can be rewritten, using Eq. (13),

$$
\left[A^{g g}\right]_{\alpha \beta}=\left(\widetilde{\boldsymbol{\phi}}_{\alpha}^{g}\right)^{T}[\widetilde{A}] \widetilde{\boldsymbol{\phi}}_{\beta}^{g},\left[A^{g \ell}\right]_{\alpha \beta}=\left(\widetilde{\boldsymbol{\phi}}_{\alpha}^{g}\right)^{T}[\widetilde{A}] \widetilde{\boldsymbol{\phi}}_{\beta}^{\ell},\left[A^{\ell \ell}\right]_{\alpha \beta}=\left(\widetilde{\boldsymbol{\phi}}_{\alpha}^{\ell}\right)^{T}[\widetilde{A}] \widetilde{\boldsymbol{\phi}}_{\beta}^{\ell},
$$

in which the $[\widetilde{A}]$ is the $(n \times n)$ matrix defined by

$$
[\widetilde{A}]=[\Phi]^{T}[\mathbb{A}][\Phi]
$$

The matrices $\left[K^{g g}\right]$ and $\left[K^{\ell \ell}\right]$ are diagonal. The generalized force is a vector in $\mathbb{C}^{n_{t}}$ which is written as $\mathcal{F}(\omega)=$ $\left(\mathcal{F}^{g}(\omega), \mathcal{F}^{\ell}(\omega)\right)$ in which $\mathcal{F}_{\alpha}^{g}(\omega)=\left(\boldsymbol{\phi}_{\alpha}^{g}\right)^{T} \mathbb{F}(\omega)$ and $\mathcal{F}_{\alpha}^{\ell}(\omega)=\left(\boldsymbol{\phi}_{\alpha}^{\ell}\right)^{T} \mathbb{F}(\omega)$. Then, for all $\omega$ fixed in $\mathcal{B}$, the generalized coordinates are calculated by solving Eq. (17) and the response $\mathbb{U}_{n_{g}, n_{\ell}}(\omega)$ is calculated using Eq. (16). 


\subsection{Subdomains decomposition of the beam-like structure domain}

The method summarized in Sections 3.1 to 3.3 is completely parameterized by the subdomains decomposition of the domain. The shape of the global eigenvectors depends on the choice of the subdomains. In [7], it is recommended to choose subdomains with a homogeneous size. This size is related to the smallest spatial wavelength which will be filtered. For a complex beam-like structure, one wants to extract the beam-like eigenvectors to construct the ROM, that is to say, we want to filter transversal local displacements. Therefore, the slender initial domain is decomposed into slices of equal thickness $\varepsilon$ of the beam-like structure (see Fig. 1).
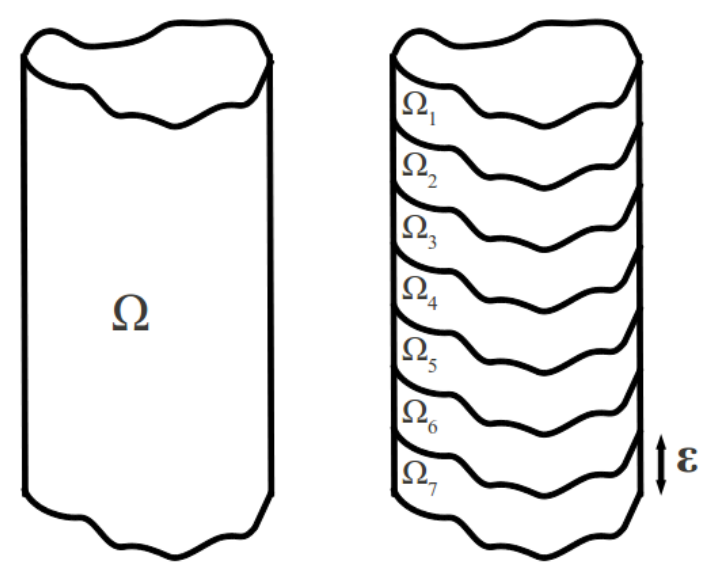

FIG. 1: Decomposition of domain $\Omega$ of the beam-like structure.

\section{UNCERTAINTY QUANTIFICATION}

In the LF range, the global elastic modes are not really sensitive to uncertainties introduced in the computational model (except uncertainties on the boundary conditions). Nevertheless, the structure under consideration had also local elastic modes in the same LF band. It is well known that the modal density of such local modes increases rapidly with the frequency and that, in addition, the local elastic modes are very sensitive to both the system-parameters uncertainties and the model uncertainties induced by the model errors. In order to improve the predictability of the reduced-order computational model in the LF range, the nonparametric probabilistic approach (see [15]) is used to take into account uncertainties in the local displacement space, that is to say, for the local generalized coordinates. The probabilistic model of uncertainties we propose is an improvement of the one introduced in [7], in which the positive-definiteness of the generalized random matrices were not guaranteed almost surely. 


\subsection{Stochastic reduced-order model}

The nonparametric probabilistic approach consists in replacing the matrices $[M],[D]$ and $[K]$ of the reduced mean matrix model defined by Eq. (17), by random matrices $[\boldsymbol{M}],[\boldsymbol{D}]$ and $[\boldsymbol{K}]$.

Let $[\mathbb{A}]$ be $[\mathbb{M}],[\mathbb{D}]$ or $[\mathbb{K}]$. Let $\boldsymbol{A}$ be $\boldsymbol{M}, \boldsymbol{D}$ or $\boldsymbol{K}$. Let $\widetilde{A}$ be $\widetilde{M}, \widetilde{D}$ or $\widetilde{K}$. Finally, let $\widetilde{\boldsymbol{A}}$ be $\widetilde{\boldsymbol{M}}, \widetilde{\boldsymbol{D}}$ or $\widetilde{\boldsymbol{K}}$. Let $E\{$. be the mathematical expectation. We introduce the random matrix $[\widetilde{\boldsymbol{A}}]$ associated with the matrix $[\widetilde{A}]=[\Phi]^{T}[\mathbb{A}][\Phi]$ (see Eq. (21)). The random matrix $[\tilde{\boldsymbol{A}}]$ is with values in the set of all the positive-definite symmetric $(n \times n)$ real matrices. Its probability density function is constructed using the maximum entropy principle under the constraints defined by the following available information: (1) the random matrix is positive definite almost surely, (2) its mean value is such that $E\{[\widetilde{\boldsymbol{A}}]\}=[\widetilde{A}]$ and (3) this random matrix verifies the following inequality $E\{\log (\operatorname{det}[\widetilde{\boldsymbol{A}}])\}=c_{A}$ with $\left|c_{A}\right|<+\infty$. The probability distribution (see [15]) of the random matrix $[\widetilde{\boldsymbol{A}}]$ depends on the mean value $[\widetilde{A}]$ and on the dispersion parameter $\delta_{A}$ which allow the level of uncertainties to be controlled and which is defined by

$$
\delta_{A}^{2}=\frac{E\left\{\|[\widetilde{\boldsymbol{A}}]-[\widetilde{A}]\|_{F}^{2}\right\}}{\|[\widetilde{A}]\|_{F}^{2}}
$$

in which $\|\cdot\|_{F}$ is the Frobenius norm. We introduce the Cholesky factorization $[\widetilde{\boldsymbol{A}}]=\left[\boldsymbol{L}_{\boldsymbol{A}}\right]^{T}\left[\boldsymbol{L}_{\boldsymbol{A}}\right]$ and $[\widetilde{A}]=$ $\left[L_{A}\right]^{T}\left[L_{A}\right]$. Then, using the double projection method, we write

$$
[\boldsymbol{A}]=\left[\begin{array}{cc}
A^{g g} & \boldsymbol{A}^{g \ell} \\
\left(\boldsymbol{A}^{g \ell}\right)^{T} & \boldsymbol{A}^{\ell \ell}
\end{array}\right],
$$

in which the block matrices are defined by

$$
\left[A^{g g}\right]_{\alpha \beta}=\left(\widetilde{\boldsymbol{\phi}}_{\alpha}^{g}\right)^{T}[\widetilde{A}] \widetilde{\boldsymbol{\phi}}_{\beta}^{g},\left[\boldsymbol{A}^{g \ell}\right]_{\alpha \beta}=\left(\widetilde{\boldsymbol{\phi}}_{\alpha}^{g}\right)^{T}\left[L_{A}\right]^{T}\left[\boldsymbol{L}_{\boldsymbol{A}}\right] \widetilde{\boldsymbol{\phi}}_{\beta}^{\ell},\left[\boldsymbol{A}^{\ell \ell}\right]_{\alpha \beta}=\left(\widetilde{\boldsymbol{\phi}}_{\alpha}^{\ell}\right)^{T}[\widetilde{\boldsymbol{A}}] \widetilde{\boldsymbol{\Phi}}_{\beta}^{\ell}
$$

It can be seen that $\left[A^{g g}\right]$ which is relative to the global generalized coordinates is deterministic, that $\left[\boldsymbol{A}^{\ell \ell}\right]$ which is relative to the local generalized coordinates is full random and that the coupling block $\left[\boldsymbol{A}^{g \ell}\right]$ is left deterministic and right random. Then, the global contributions which are not really sensitive to uncertainties are kept deterministic. The random matrix $[\boldsymbol{A}]$ can be written as $[\boldsymbol{A}]=\left[L_{A} \widetilde{\boldsymbol{\phi}}^{g} \boldsymbol{L}_{\boldsymbol{A}} \widetilde{\boldsymbol{\phi}}^{\ell}\right]^{T}\left[L_{A} \widetilde{\boldsymbol{\phi}}^{g} \boldsymbol{L}_{\boldsymbol{A}} \widetilde{\boldsymbol{\phi}}^{\ell}\right]$. It can then be deduced that random matrix $[\boldsymbol{A}]$ is positive definite almost surely. 


\subsection{Random frequency responses}

The random response $\boldsymbol{U}_{n_{g}, n_{l}}(\boldsymbol{\omega})$ is then written as

$$
\boldsymbol{U}_{n_{g}, n_{\ell}}(\boldsymbol{\omega})=\sum_{\alpha=1}^{n_{g}} Q_{\alpha}^{g}(\boldsymbol{\omega}) \boldsymbol{\phi}_{\alpha}^{g}+\sum_{\beta=1}^{n_{\ell}} Q_{\beta}^{\ell}(\omega) \boldsymbol{\phi}_{\beta}^{\ell},
$$

in which the random vector $\boldsymbol{Q}(\omega)=\left(\boldsymbol{Q}^{g}(\omega), \boldsymbol{Q}^{\ell}(\omega)\right)$ with values in $\mathbb{C}^{n_{t}}$ of all the random generalized coordinates is such that $\boldsymbol{Q}^{g}(\boldsymbol{\omega})=\left(Q_{1}^{g}(\omega), \ldots, Q_{n_{g}}^{g}(\omega)\right)$ and $\boldsymbol{Q}^{\ell}(\omega)=\left(Q_{1}^{\ell}(\omega), \ldots, Q_{n_{\ell}}^{\ell}(\omega)\right)$. Consequently, the random vector $\boldsymbol{Q}(\omega)$ is solution of the following stochastic reduced matrix equation,

$$
\left(-\omega^{2}[\boldsymbol{M}]+i \omega[\boldsymbol{D}]+[\boldsymbol{K}]\right) \boldsymbol{Q}(\omega)=\mathcal{F}(\omega)
$$

This equation is solved using the Monte Carlo simulation method [16].

It should be noted that the probabilistic model of uncertainties is implemented in the ROM. The computation of the global eigenvectors and the local eigenvectors of the dynamical model is carried out outside the Monte Carlo loop. Consequently, as soon as the mean ROM (see Eq. (17)) is constructed, then the stochastic ROM is deduced (see Eq. (26)) and each Monte Carlo loop is relatively fast. Furthermore, the Monte Carlo simulation can efficiently be implemented as a parallel Monte Carlo solver. The stochastic ROM is not of small size if the modal density is very high, and particularly there is no size gain with respect to a classical modal analysis. For instance, a real fuel assembly of pressurized water reactor has more than 10,000 elastic modes in the LF band due to the numerous local modes of each fuel rod. The interest of the approach lies in the separation between the global contributions and the local contributions which allows the global displacements and the local displacements to be modeled in different ways with respect to uncertainties.

\section{APPLICATION TO FUEL ASSEMBLIES}

In this section, we apply and validate the methodology to a computational model of a half fuel assemblies.

\subsection{Mean computational model}

The dynamical system is made up of 49 tubes linked each to the others by four grids. There are two types of tubes: (1) the guide-tubes (black tubes on Fig. 2) which are clamped at their ends and welded to the grids and (2) the fuel- 

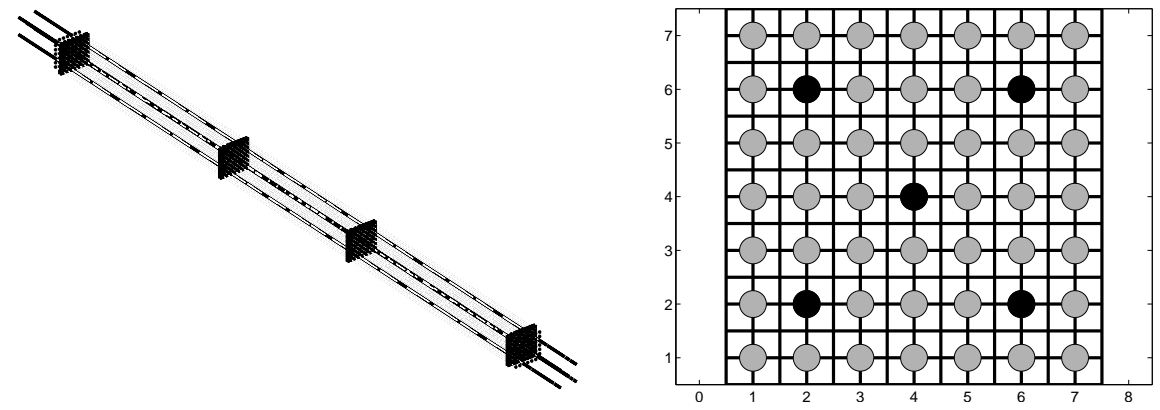

FIG. 2: Geometry of the dynamical system. Left figure: 3D-Mesh. Right figure: Grid-view showing 5 guide-tubes (black disks) and 44 fuel-tubes (gray disks).

tubes (gray tubes on Fig. 2) which are free at their ends and linked to the grids by linear springs. The guide-tubes are circular, homogeneous, isotropic beam with constant outer radius $6.0 \times 10^{-3} \mathrm{~m}$, thickness $4.0 \times 10^{-4} \mathrm{~m}$, length $2.25 \mathrm{~m}$, mass density $6526 \mathrm{~kg} / \mathrm{m}^{3}$, Poisson ratio 0.3 , Young modulus $9.84 \times 10^{10} \mathrm{~N} / \mathrm{m}^{2}$. The fuel-tubes are circular, homogeneous, isotropic beam with constant outer radius $4.75 \times 10^{-3} \mathrm{~m}$, thickness $5.7 \times 10^{-4} \mathrm{~m}$, length $1.91 \mathrm{~m}$, mass density $3.79 \times 10^{4} \mathrm{~kg} / \mathrm{m}^{3}$ (equivalent mass density), Poisson ratio 0.3 , Young modulus $9.84 \times 10^{10} \mathrm{~N} / \mathrm{m}^{2}$. The four grids are assemblages of rectangular, homogeneous, isotropic beams with constant height $2.7 \times 10^{-2} \mathrm{~m}$, thickness $4.8 \times 10^{-4} \mathrm{~m}$, mass density $1.2 \times 10^{4} \mathrm{~kg} / \mathrm{m}^{3}$, Poisson ratio 0.3 , Young modulus $9.84 \times 10^{10} \mathrm{~N} / \mathrm{m}^{2}$. The guide-tube/grid springs have a stiffness equal to $5.0 \times 10^{9} \mathrm{~N} / \mathrm{m}$ for the three translations and $5.0 \times 10^{6} \mathrm{~N} / \mathrm{m}$ for the three rotations. The fuel-tube/grid springs have stiffness equal to $1.8 \times 10^{5} \mathrm{~N} / \mathrm{m}$ for the three translations and $92 \mathrm{~N} / \mathrm{m}$ for the three rotations. The frequency band of analysis is $\mathcal{B}=2 \pi \times] 0,120] \mathrm{rad} / \mathrm{s}$. The mean computational model is made up of Timoshenko beam elements and linear spring elements. The structure has $m=12,750$ DOF.

\subsection{Modal analysis, global and local eigenvectors}

In a first step, the elastic modes are calculated with the mean computational model defined by Eq. (2). There are 447 eigenfrequencies in the frequency band of analysis $\mathcal{B}$ and $n=500$ eigenfrequencies in the band $2 \pi \times] 0,147.3] \mathrm{rad} / \mathrm{s}$. The $4^{\text {th }}$ elastic mode $\varphi_{4}$ and the $10^{\text {th }}$ elastic mode $\varphi_{10}$ are displayed in Fig. 3 . We can see that $\boldsymbol{\varphi}_{4}$ is a global elastic mode while $\boldsymbol{\varphi}_{10}$ is a local elastic mode. In a second step, the global eigenvectors and the local eigenvectors are computed. In order to construct the matrix $\left[H^{r}\right]$, the domain is split into 49 subdomains which correspond to 49 longitudinal slices of the structure domain. In the band $] 0,147.3] \mathrm{Hz}$, there are $n_{g}=23$ global eigenvectors and $n_{\ell}=$ 477 local eigenvectors. Figure 4 displays the distribution of the number of eigenvalues for the global eigenvectors and for the local eigenvectors. The eigenvalues $\lambda^{g}$ and $\lambda^{\ell}$ are expressed in terms of frequency as $\sqrt{\lambda^{g}} /(2 \pi)$ and $\sqrt{\lambda^{\ell}} /(2 \pi)$. 


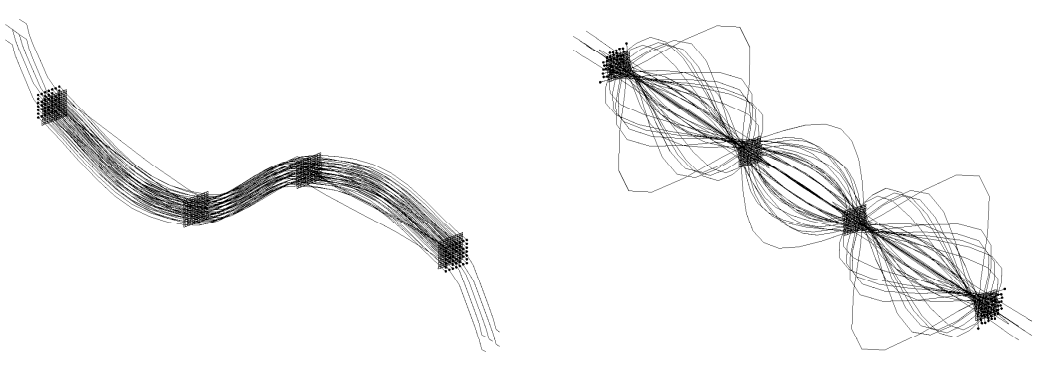

FIG. 3: $4^{\text {th }}$ elastic mode (left) and $10^{\text {th }}$ elastic mode (right).

It can be seen that there are numerous local eigenvectors intertwined with the global eigenvectors.

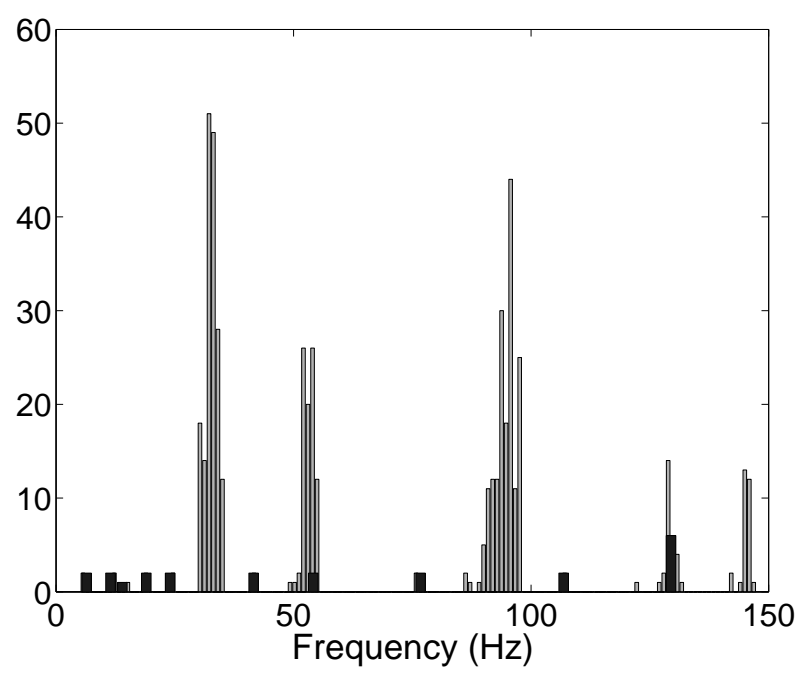

FIG. 4: Distribution of the number of eigenvalues for the global eigenvectors (black histogram) and for the local eigenvectors (gray histogram) as a function of the frequency

\subsection{Frequency responses calculated with the reduced-order model}

For all $\omega$ in $\mathcal{B}$, the structure is subjected to two external point loads equal to $1 N$ applied to a node which belongs to the lowest grid (stiff part) and a node belonging to the fuel-tube 3-3 (see Fig. 2) (flexible part) located between the two lowest grids. The mean damping matrix is constructed using a modal damping corresponding to a damping rate $\xi=0.01$. The response is calculated at two observation points, the point Pobs $_{1}$ located in the highest grid (stiff part) and the point $\mathrm{Pobs}_{2}$ belonging to the fuel-tube 3-3 (flexible part) located between the two highest grids. The response is calculated for different projections associated with the different bases: the elastic modes with Eq. (3) $(n=500)$, the 
global eigenvectors with Eq. (16) $\left(n_{g}=23\right.$ and $\left.n_{\ell}=0\right)$, the local eigenvectors with Eq. (16) $\left(n_{g}=0\right.$ and $\left.n_{\ell}=477\right)$ and finally, both the global and the local eigenvectors with Eq. (16) $\left(n_{g}=23\right.$ and $\left.n_{\ell}=477\right)$. The modulus of the responses are displayed in Fig. 5. It can be seen that the responses calculated using both the global and the local
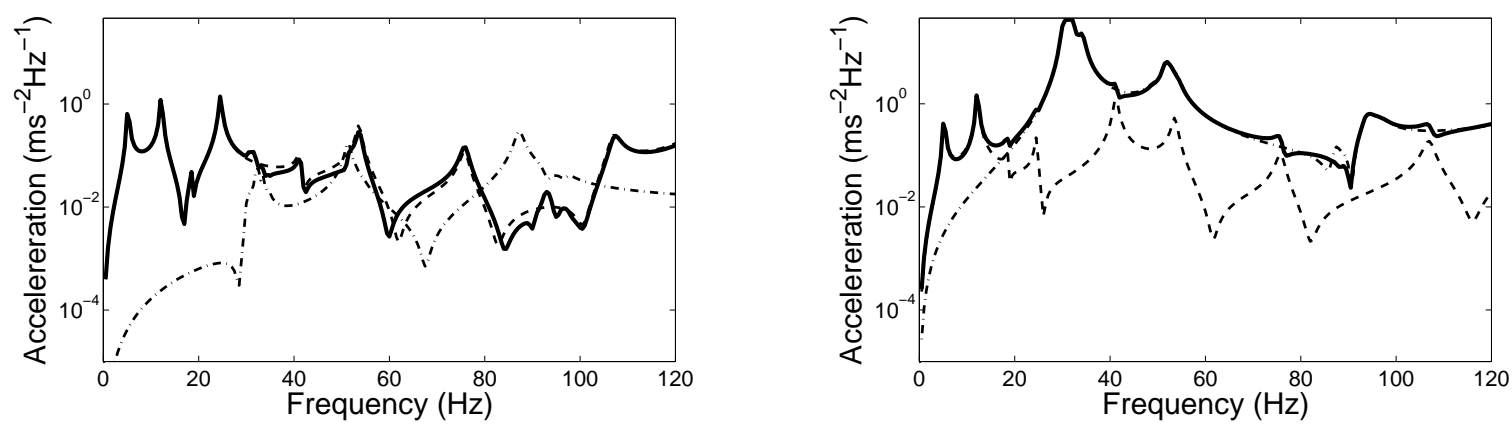

FIG. 5: Frequency response functions in $\log$ scale for $\mathrm{Pobs}_{1}$ (left) and $\mathrm{Pobs}_{2}$ (right). Comparisons between different projection bases: the elastic modes (thick solid line), the global eigenvectors only (dashed line), the local eigenvectors only (mixed line), both the global and the local eigenvectors (thin solid line superimposed to the thick solid line).

eigenvectors are exactly the same that the response calculated using the elastic modes. For point Pobs ${ }_{1}$ in the stiff part, the contribution of the global eigenvectors is preponderant. For point Pobs $_{2}$ in the flexible part, the contribution of the local eigenvectors is important except for the two first resonances corresponding to the first global eigenvectors.

\subsection{Random frequency responses calculated with the stochastic reduced-order model}

The random frequency responses are calculated as explained in Section 4.2. The dispersion parameters are chosen as $\delta_{M}=0.1, \delta_{D}=0.0$ and $\delta_{K}=0.1$. The Monte Carlo simulation method is carried out with 400 simulations. The confidence regions corresponding to a probability level $P_{c}=0.98$ are presented in Fig. 6 . It can be seen that for observation points $\mathrm{Pobs}_{1}$ and $\mathrm{Pobs}_{2}$ the sensitivity of the resonances relative to the global eigenvectors with respect to uncertainties is low. This variability increases at the frequencies for which the local contributions are not negligible. For this application, we have constructed the stochastic ROM in order to carry out a sensitivity analysis of the structure with respect to uncertainties. It should be noted that if experimental responses were available, the dispersion parameters could be identified experimentally by solving an inverse stochastic problem (see [17]) .

\section{CONCLUSIONS}

A general method has been developed and validated to construct a stochastic reduced-order model for complex beamlike dynamical structure having a high modal density in the LF range. The projection basis is made up of two families 

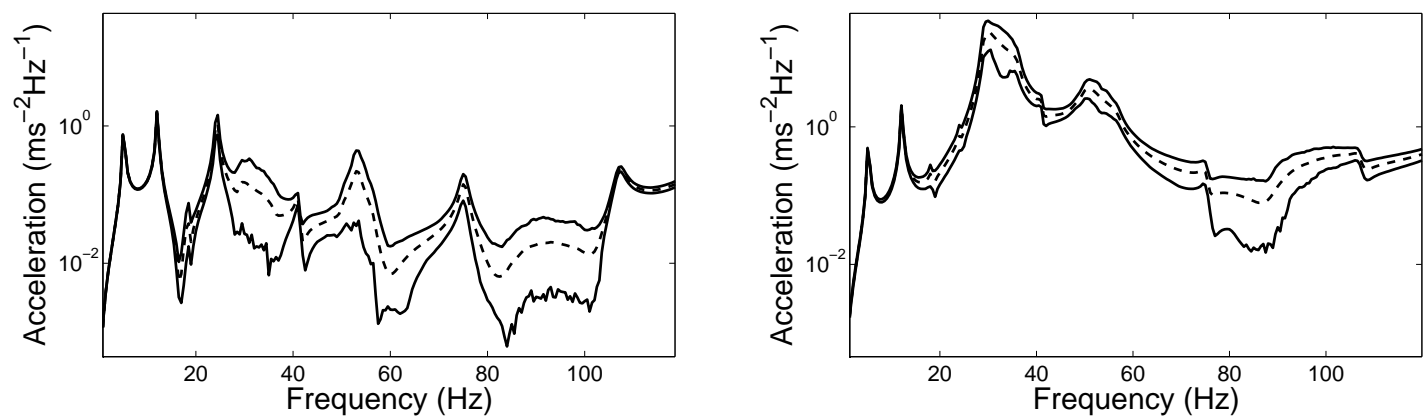

FIG. 6: Random frequency response functions for points Pobs 1 (left) and $\mathrm{Pobs}_{2}$ (right). Confidence region (lower and upper lines), mean response (dashed line).

of vectors: the global eigenvectors and the local eigenvectors which are separately computed. This separation allows a probabilistic model of uncertainties to be implemented only in the subspace of admissible displacements spanned by the local eigenvectors. Then the stochastic reduced-order model can be used to carry out a sensitivity analysis of the dynamical response with respect to uncertainties. If experimental responses were available, then the dispersion parameters could be identified as a statistical inverse problem.

\section{REFERENCES}

1. Craig, R. and Kurdila, A., Fundmentals of Structural Dynamics, John Wiley and Sons, New Jersey, 2nd edition, 2006.

2. Ohayon, R. and Soize, C., Structural Acoustics and Vibration, Academic Press, San Diego, 1998.

3. Ewins, D., Modal Testing: theory and practice, Research Study Press LTD, John Wiley and Sons, 1984.

4. Soize, C., Capiez-Lernout, E., and Ohayon, R., Robust updating of uncertain computational models using experimental modal analysis, AIAA Journal, 46(11):2955-2965, 2008.

5. Soize, C., Reduced models in the medium frequency range for general dissipative structural-dynamics systems, European Journal of Mechanics, A/Solids, 17(4):657-685, 1998.

6. Soize, C., Probabilistic structural modeling in linear dynamic analysis of complex mechanical systems, I - Theoretical elements, La Recherche Aérospatiale (English edition), 5:23-48, 1986.

7. Soize, C. and Batou, A., Stochastic reduced-order model in low-frequency dynamics in presence of numerous local elastic modes, Journal of applied mechanics - Transactions of the ASME, 78(6):061003, 2011. 
8. Noor, A., Anderson, M., and Greene, W., Continuum models for beam- and platelike-lattice structures, AIAA Journal, 16(12):1219-1228, 1978.

9. Planchard, J., Vibrations of nuclear fuel assemblies: a simplified model, Nuclear engineering and design, 86(3):383-391, 1995.

10. Sigrits, J. and Broc, D., Dynamic analysis of a tube bundle with fluid-structure interaction modelling using a homogenisation method, Computer Methods in Applied Mechanics and Engineering, 197(9-12):1080-1099, 2008.

11. Langley, R. and Bremmer, P., A hybrid method for the vibration analysis of complex structural-acoustic systems, Journal of the Acoustical Society of America, 105(3):1657-1672, 1999.

12. Karhunen, K., Zur spektraltheorie stochasticher prozesse, Ann. Acad. Sci. Fennicae, 220, 1945.

13. Loève, M., Probability Theory, Van Nostrand, New York, 3rd edition, 1963.

14. Holmes, P., Lumley, J., and Berkooz, G., Turbulence, Coherent Structures, Dynamical Systems and Symmetry, Cambridge University Press, Cambridge, 1997.

15. Soize, C., Random matrix theory for modeling random uncertainties in computational mechanics, Computer Methods in Applied Mechanics and Engineering, 194(1216):1333-1366, 2005.

16. Rubinstein, R., Simulation and the Monte Carlo Method, John Wiley and Sons, 1980.

17. Batou, A. and Soize, C., Experimental identification of turbulent fluid forces applied to fuel assemblies using an uncertain model and fretting-wear estimation, Mechanical Systems and Signal Processing, 27(7):2141-2153, 2009. 